\title{
Desordem temporomandibular (DTM): relato de caso de mioespasmo (mialgia de contração tônica)
}

\author{
Temporomandibular disorder (TMD): \\ case report of myospasm (tonic contraction myalgia) \\ Desorden temporomandibular (DTM): \\ reporte de caso de mioespasmo (mialgia de contracción tónica)
}

Helinaldo Corrêa da CONCEIÇÃO ${ }^{1}$

Dannilo Wiklymber Roldao MENDONÇA ${ }^{1}$

Dionísio Leão DOCE ${ }^{1}$

Gleicy Gomes de LIMA ${ }^{\mathbf{1}}$

Maria de Fátima ARAÚJO²

Vilma da Silva MELO

Lioney Nobre CABRAL ${ }^{3}$

Tiago Novaes PINHEIRO ${ }^{4}$

${ }^{1}$ Acadêmico do Curso de Graduação em Odontologia, Escola Superior de Ciências da Saúde,

Universidade do Estado do Amazonas, UEA, 69065-001 Manaus-AM, Brasil

${ }_{2}^{2}$ Doutoranda em Saúde Coletiva, Instituto de Medicina Social, Universidade do Estado do Rio de Janeiro, IMS-UERJ, 20550-013 Rio de Janeiro - RJ, Brasil

${ }^{3}$ Professor da Escola Superior de Ciências da Saúde, Universidade do Estado do Amazonas, UEA, 69065-001 Manaus-AM, Brasil. Doutor em Biotecnologia pela Universidade Federal do Amazonas, UFAM

${ }^{4}$ Professor da Escola Superior de Ciências da Saúde, Universidade do Estado do Amazonas, UEA, 69065-001 Manaus-AM, Brasil. Mestre e Doutor em Patologia Bucal pela Faculdade de Odontologia de Bauru, Universidade de São Paulo, USP

\section{Resumo}

O mioespasmo é uma alteração involuntária sustentada por mediação central. É uma alteração em que o músculo permanece contraído por alguns segundos, minutos ou mesmo dias e também é frequentemente observado durante tratamento em que necessitam de sessões prolongadas, como, por exemplo, tratamentos endodônticos de molares. Depois de determinado tempo, o paciente mostra instabilidade para manter a boca aberta, apresentando episódios de fechamento repentinos. Mioespasmos são facilmente reconhecidos pela disfunção estrutural que produzem. Descrever o diagnóstico de um caso de mioespasmo em paciente com mordida aberta anterior, otalgia e dor na própria ATM. A paciente M.M.S.R, 33 anos, gênero feminino, cor parda, compareceu ao atendimento na Policlínica da UEA para exame estomatológico de rotina. Durante a anamnese a paciente relatou que sentia dores de cabeça, dor de ouvido, apertamento dos dentes durante a noite e possuía o elemento 46 fraturado, relatou também ter ido ao dentista fazer a restauração do elemento dental 46 há aproximadamente um ano e que, passado alguns dias do procedimento começou a ter dores de cabeça frequentemente, porém a paciente relatou ser muito estressada. Por conseguinte, a etiologia dos mioespasmos não tem sido bem documentada. É provável que muitos fatores se combinem para promover o mioespasmo. Condições musculares locais certamente aparecem para criar mioespasmos: as dores de cabeça, de ouvido, dor facial, má oclusão aguda, dentre outros. Compete ao cirurgião-dentista diagnosticar e tratá-las em conjunto com outras especialidades.

Descritores: Espasmo; Mialgia; Mordida Aberta; Otite; Transtornos da Articulação Temporomandibular.

\section{Abstract}

Myospasm is an involuntary alteration sustained by central mediation. It is a change in which the muscle remains seconds, minutes or even days and is also often observed during treatment requiring prolonged sessions, such as endodontic molar treatments. After some time, the patient shows instability to keep his mouth open, presenting sudden closure episodes. Myospasms are easily recognized by the structural dysfunction they produce. To describe the diagnosis of a case of myospasm in a patient with anterior open bite, otalgia and pain in the TMJ itself. The patient M.M.S.R, 33 years old, female, brown, attended the service in the UEA is polyclinic for routine stomatological examination. During the anamnesis the patient reported that she had headaches, earache, clenching of the teeth at night and had the fractured element 46, also reported having gone to the dentist to restore the dental element 46 about a year ago and that, a few days of the procedure began to have headaches frequently, but the patient reported being very stressed. Therefore, the etiology of myospasm has not been well documented. Many factors are likely to combine to promote myospasm. Local muscle conditions certainly appear to create myospasms: headaches, earaches, facial pain, acute malocclusion, among others. It is up to the dental surgeon to diagnose and treat them together with other specialties.

Descriptors: Spasm; Myalgia; Open Bite; Otitis; Temporomandibular Joint Disorders.

\section{Resumen}

El mioespasmo es una alteración involuntaria sostenida por mediación central. Es un cambio en que el músculo permanece contraído por algunos segundos, minutos o incluso días y también es frecuentemente observado durante el tratamiento en que necesitan sesiones prolongadas, como, por ejemplo, tratamiento endodóntico de molares. Después de cierto tiempo, el paciente muestra inestabilidad para mantener la boca abierta, presentando episodios de cierre repentinos. Los mioespasmos son fácilmente reconocidos por la disfunción estructural que producen. Describir el diagnóstico de un caso de mioespasmo en paciente con mordida abierta anterior, otalgia y dolor en la propia ATM. La paciente M.M.S.R, 33 años, género femenino, color pardo, asistió a la atención en la Policlínica de la UEA para examen estomatológico de rutina. Durante la anamnesis la paciente relató que sentía dolores de cabeza, dolor de oído, apretón de los dientes durante la noche y poseía el elemento 46 fracturado, relató también haber ido al dentista a hacer la restauración del elemento dental 46 hace aproximadamente un año y que, pasado algunos días del procedimiento comenzó a tener dolores de cabeza a menudo, pero la paciente relató ser muy estresada. Por lo tanto, la etiología de los mioespasmos no ha sido bien documentada. Es probable que muchos factores se combinen para promover el mioespasmo. Las condiciones musculares locales ciertamente aparecen para crear mioespasmos: los dolores de cabeza, de oído, dolor facial, mala oclusión aguda, entre otros. Compete al cirujano-dentista diagnosticar y tratarlas en conjunto con otras especialidades.

Descriptores: Espasmo; Mialgia; Mordida Abierta; Otitis; Trastornos de la Articulación Temporomandibular.

\section{INTRODUÇÃO}

Mioespasmo é uma contração muscular tônica induzida pelo sistema nervoso central (SNC), ou seja, dor musculoesquelética. Mioespasmo não são crônicos, ao contrário representam uma condição de duração relativamente curta. Durante muitos anos os cientistas achavam que os mioespasmos eram a fonte mais comum de dor miogênica ${ }^{1-3}$. Estudos mais recentes, entretanto, deram nova luz sobre as dores musculares e mioespasmos. É razoável 
suspeitar que um músculo em espasmo ou contração tônica poderia revelar um nível relativamente alto de atividade eletromiográfica (EMG). Os estudos, entretanto, não apoiam a suposição de que músculos doloridos têm um significante aumento na sua atividade $\mathrm{EMG}^{4-8}$.

$\mathrm{O}$ mioespasmo se apresenta como uma das causas à abertura prolongada da boca. De etiologia pouco documentada, pode ser definido como um conjunto de distúrbios articulares e musculares que acomete a região orofacial e músculos da mastigação, provocando disfunção estrutural. Acredita-se que condições musculares locais que envolvam fadiga muscular e mudanças no equilíbrio eletrolítico local, assim como estímulos de dor profunda atuem no desenvolvimento do quadro, o qual geralmente envolve má oclusão em decorrência das alterações de posição mandibular ${ }^{9-11}$.

As contrações musculares clássicas do mioespasmo podem durar minutos e até dias. Clinicamente, observa-se limitação da abertura bucal e má oclusão aguda, dependendo do músculo acometido. Quando o paciente apresenta limitação da abertura bucal associado à dor na ATM, podem estar presentes dois problemas: espasmo do masseter e deslocamento anterior do disco sem redução em fase aguda. $\mathrm{O}$ tratamento consiste primeiramente em identificar os agentes causais ou predisponentes. Após, a identificação do problema, lança-se mão de terapêuticas à base de: analgésicos, calor local, massagem, exercícios, estimulação elétrica transcutânea, acupuntura, dispositivos interoclusais (no apertamento ou bruxismo), bloqueio muscular anestésico sem vaso constritor todos esses métodos são medidas que podem produzir algum alívio da dor. Em fase aguda, pode-se empregar frio sob a forma de spray refrigerante ${ }^{12}$.

Os fatores que precipitam ou agravam a dor do paciente são de importância fundamental no diagnóstico. Os fatores agravantes não só sugerem os tipos de tecidos que podem estar envolvidos, mas também auxiliam no direcionamento de testes objetivos. É importante ser específico ao registrar as informações. Se o paciente relata dor quando se alimenta, tenham em mente que várias estruturas são estimuladas durante a mastigação, como músculos, articulações temporomandibulares (ATMs), mucosa e potencialmente a polpa. Seja específico quanto ao fator agravante. A falta de um fator agravante é uma indicação de que a dor não é de origem odontogênica ${ }^{13}$.

De um modo geral, a maior parte dos sintomas de desordem temporomandibular (DTM) é relatada pela população de 19 a 45 anos, em uma relação de oito mulheres para cada caso relatado em homem. Geralmente a abordagem terapêutica é multidisciplinar no gênero feminino. Aproximadamente $33 \%$ da população tem pelo menos um sintoma de DTM e em cerca de 3 a $7 \%$ dos casos a gravidade é suficiente para o paciente procurar tratamento ${ }^{14,15}$.

A divulgação deste caso clínico é de grande importância no meio científico e social, servindo como base para direcionar o tratamento em situações semelhantes, visto que trabalhos versando sobre mioespasmo são pouco relatados na literatura científica.

\section{CASO CLÍNICO}

A estudante de Direito M.M.S.R, 33 anos, gênero feminino, cor parda, compareceu para atendimento a Policlínica da Universidade Estadual do Amazonas,UEA para exame semiológico de rotina. Durante a anamnese, a paciente relatou que sentia dores de cabeça, dor de ouvido, apertamento dos dentes durante a noite e possuía o elemento 46 fraturado. Relatou também ter ido ao dentista fazer a restauração do elemento dental há aproximadamente um ano e que, passado alguns dias do procedimento começou a ter dores de cabeça frequentemente, porém a paciente relatou ser muito estressada. Ao exame extrabucal constatou-se a presença de mordida aberta anterior e mordida cruzada posterior. (Figura 1), havia alterações nos músculos esternocleidomastóideo (feixe esternal superficial e clavicular profundo), temporal feixe anterior, dor na articulação temporomandibular (ATM), bem como dores otológicas. Foram medidas a lateralidade direita $(6 \mathrm{~mm})$ (Figura 2), lateralidade esquerda $(1 \mathrm{~mm})$ (Figura 3$)$, protrusão $(10 \mathrm{~mm})$ (Figura 4) e abertura máxima (35 mm) (Figura 5). Sobre a qualidade do sono a paciente relatou sono leve, com episódios de insônia. Ao exame exame intrabucal detectou-se o elemento 46 fraturado e com extensa restauração de resina composta na oclusal (Figura 6).

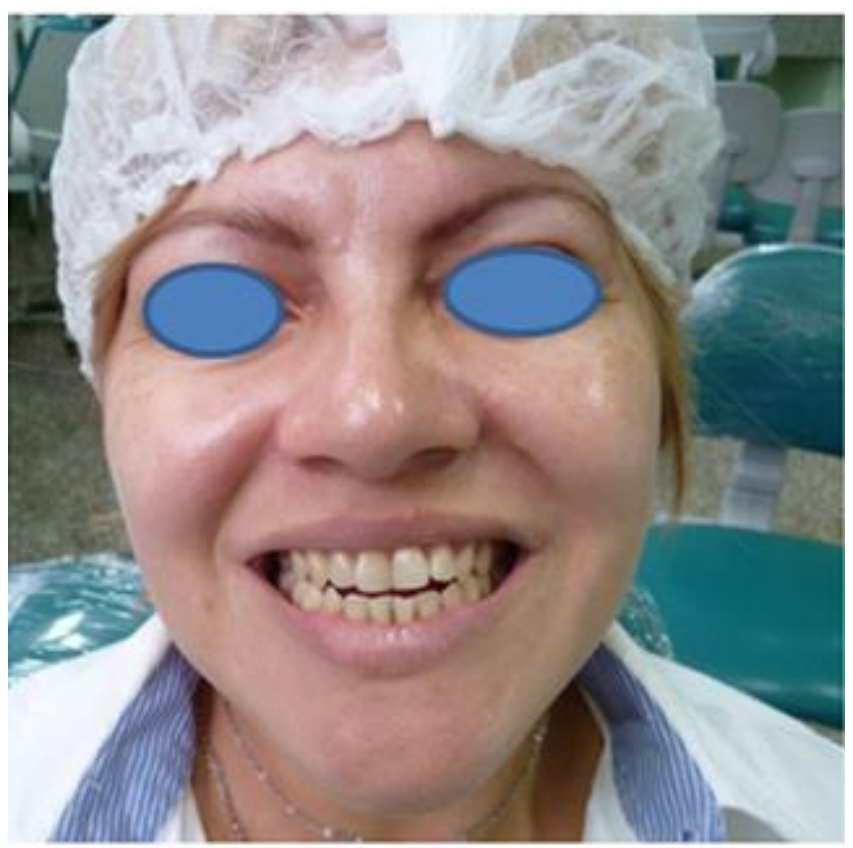

Figura 1: Presença de mordida aberta anterior e mordida cruzada posterior.

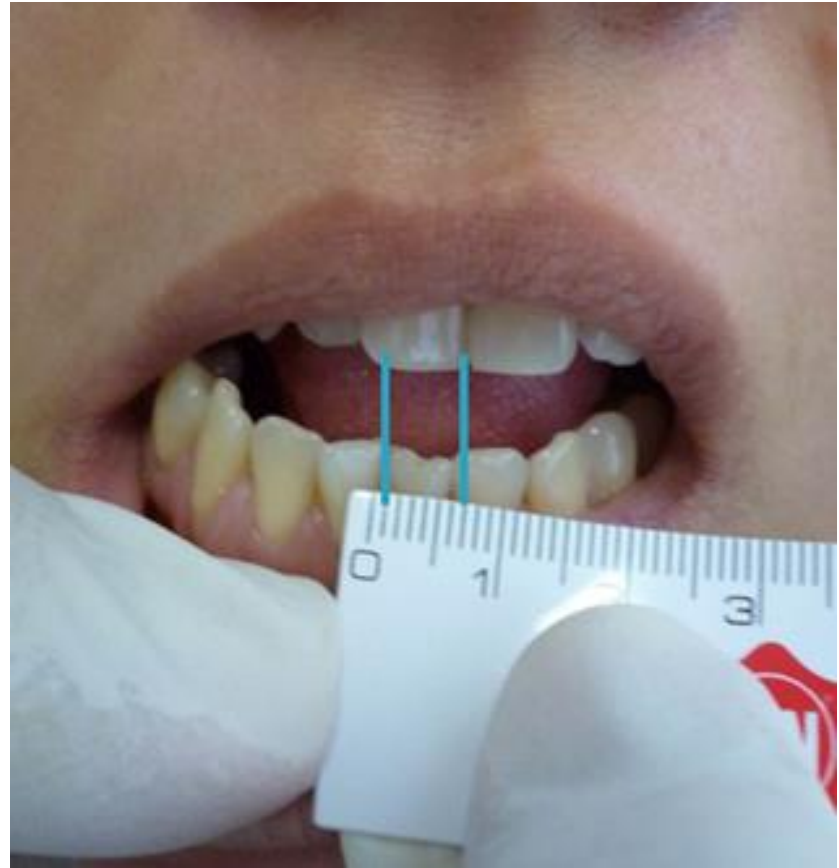

Figura 2: Medida da lateralidade direita (6mm).

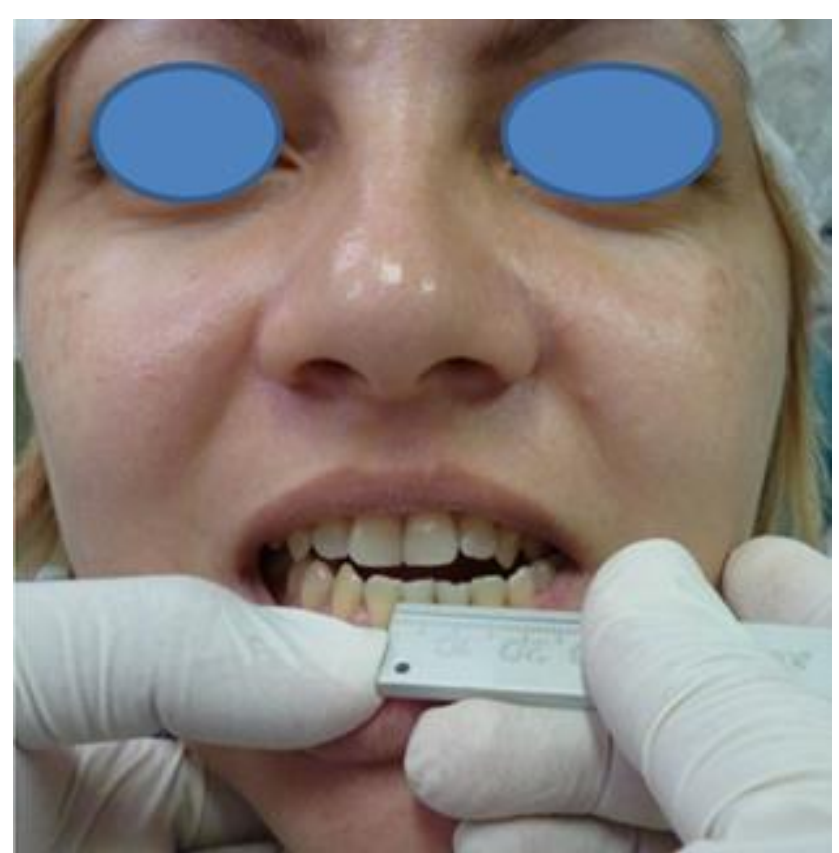

Figura 3: Medida da lateralidade esquerda (1mm) 


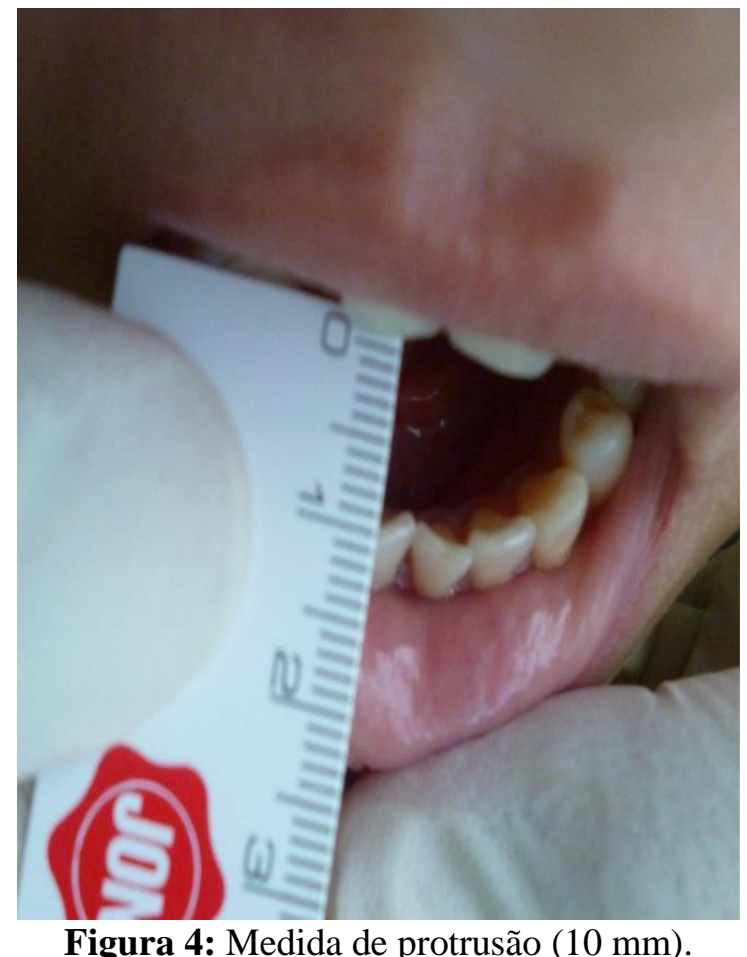

Figura 4: Medida de protrusão $(10 \mathrm{~mm})$.

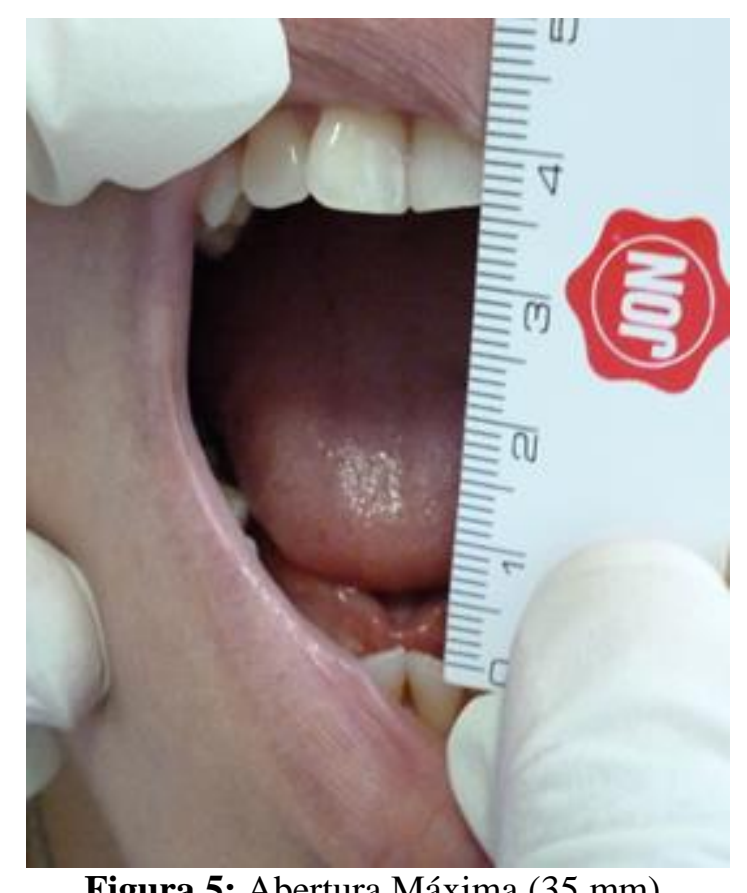

Figura 5: Abertura Máxima (35 mm).

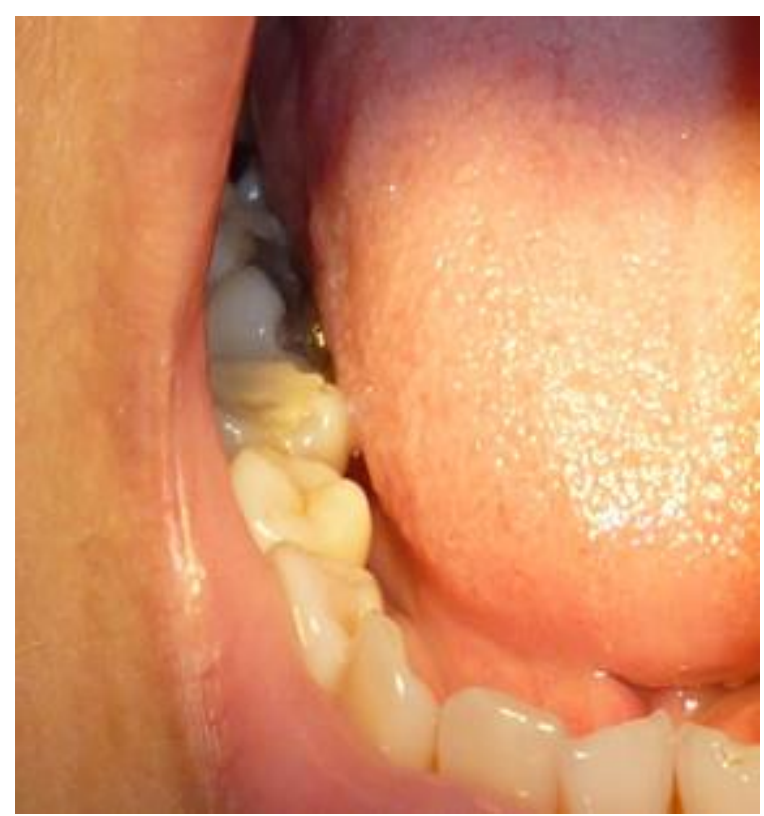

Figura 6: Extensa restauração de resina composta no elemento 46.

Realizado o exame de Tomografia Computadorizada de Feixe Cônico (TCFC) com o paciente com a boca fechada em oclusão, em máxima intercuspidação, para avaliação dos demais elementos dentários e da articulação temporomandibular (ATM). Foi constatada perda óssea na região de furca do elemento 46 como também perda óssea em elementos adjacentes. Pela TCFC foi detectada compressão do disco articular do lado direito, com medida do espaço articular de $12 \mathrm{~mm}$ (Figuras 7 e 8) contra a média de $23 \mathrm{~mm}$ para o espaço normal. Para o lado esquerdo observou-se valor de $26 \mathrm{~mm}$ (Figuras 9 e 10), dentro dos parâmetros de normalidade.

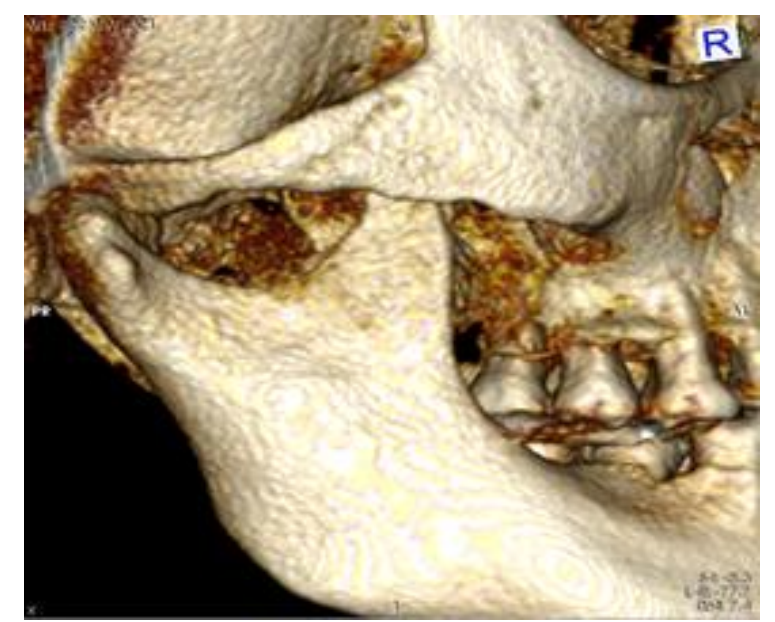

Figura 7: Espaço articular lado direito $0,12 \mathrm{~cm}$.

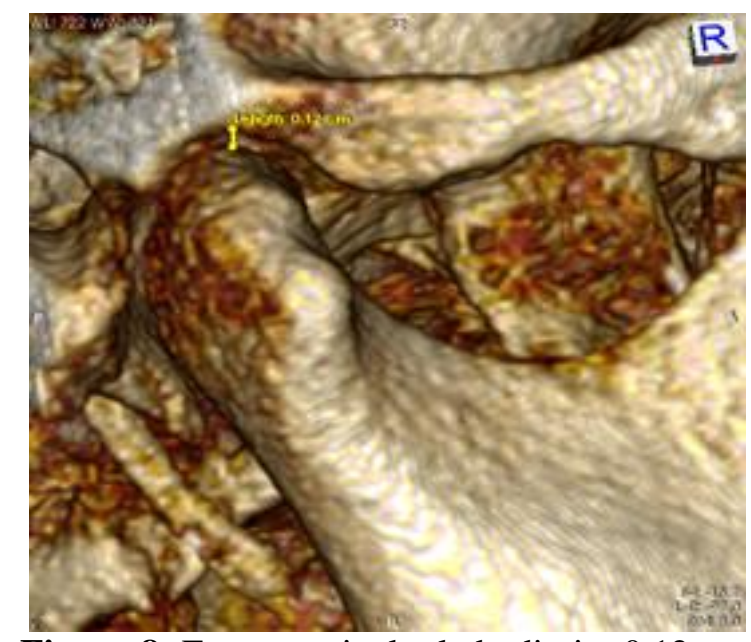

Figura 8: Espaço articular lado direito $0,12 \mathrm{~cm}$

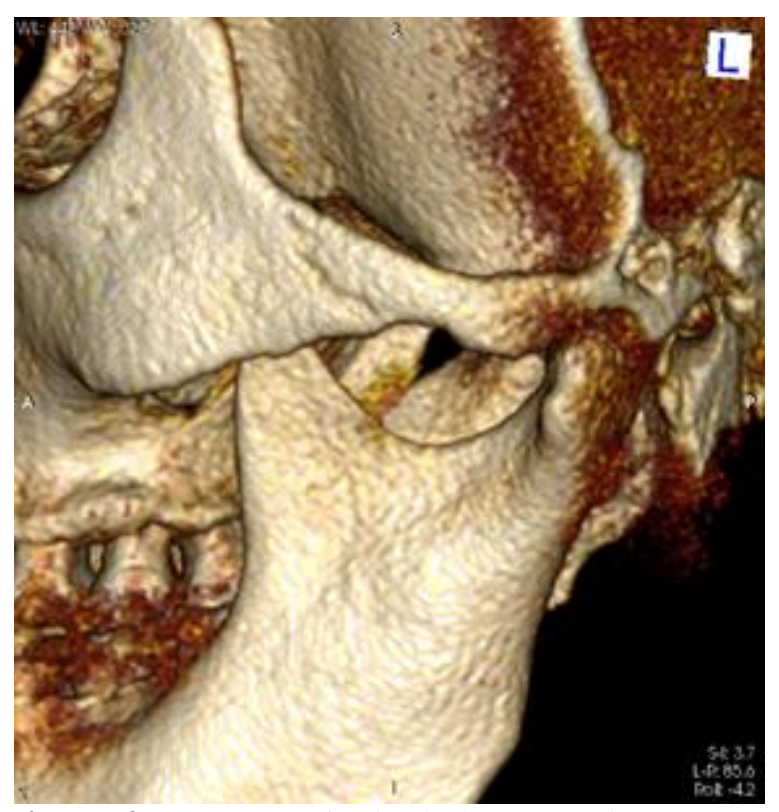

Figura 9: Espaço articular lado esquerdo $0,26 \mathrm{~cm}$

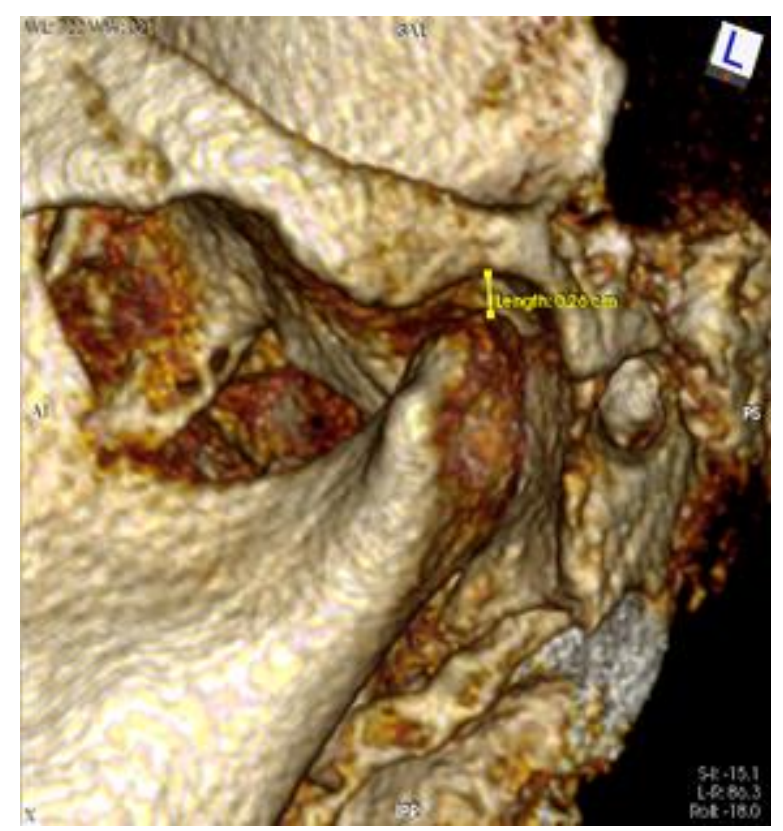

Figura 10: Espaço articular lado esquerdo $0,26 \mathrm{~cm}$

A partir da conclusão dos exames complementares tiveram início os procedimentos clínicos. Assim, foram prescritos cinco exercícios fisioterápicos, com dez repetições cada, duas vezes ao dia, pela manhã e à noite (Figuras $11 \mathrm{e}$ 12). 


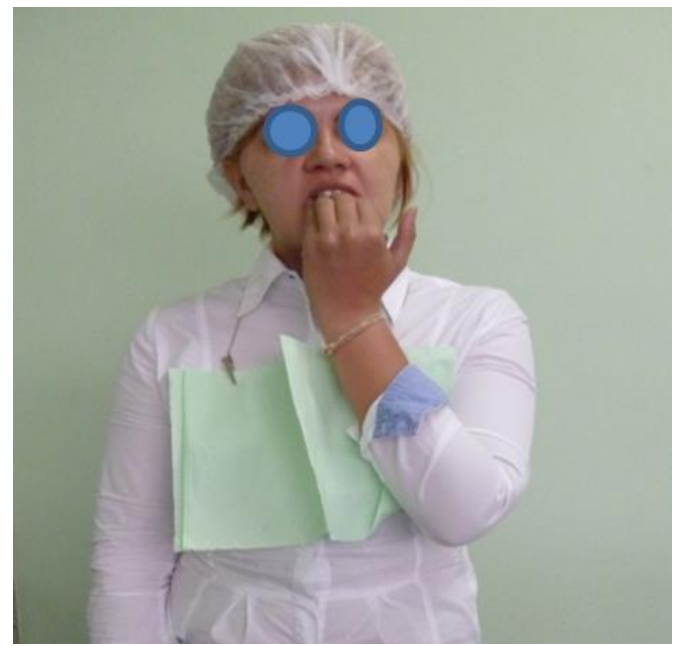

Figura 11: Exercício fisioterápico.

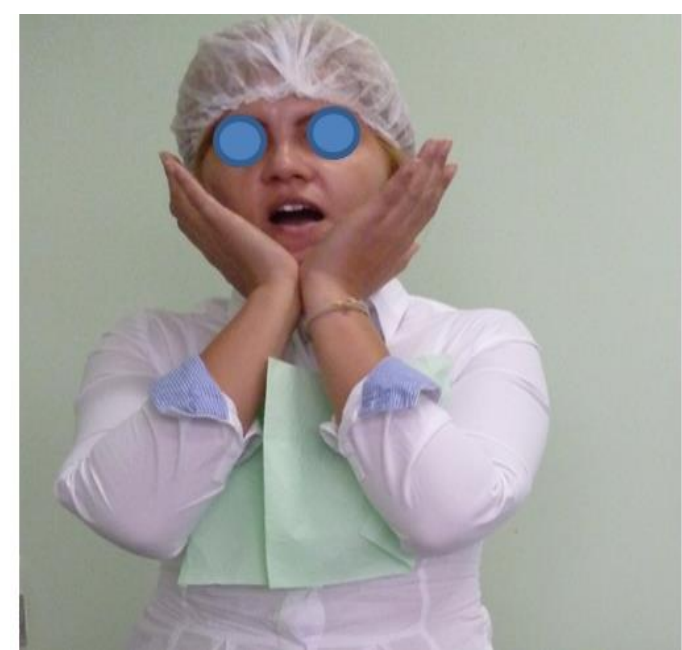

Figura 12: Exercício fisioterápico.

Não foi feita placa miorelaxante porque a paciente já fazia uso de uma e era condizente com o tratamento. Foi sugerida raspagem subgengival e supragengival, tanto na arcada inferior como na superior devido à presença de cálculo dentário e também restauração do elemento 46 com resina composta, já que a paciente recusava autorizar exodontia do elemento dentário. Duas semanas após o início do tratamento, o movimento de lateralidade esquerda mostrou-se ampliado, passando de $1 \mathrm{~mm}$ para $9 \mathrm{~mm}$.

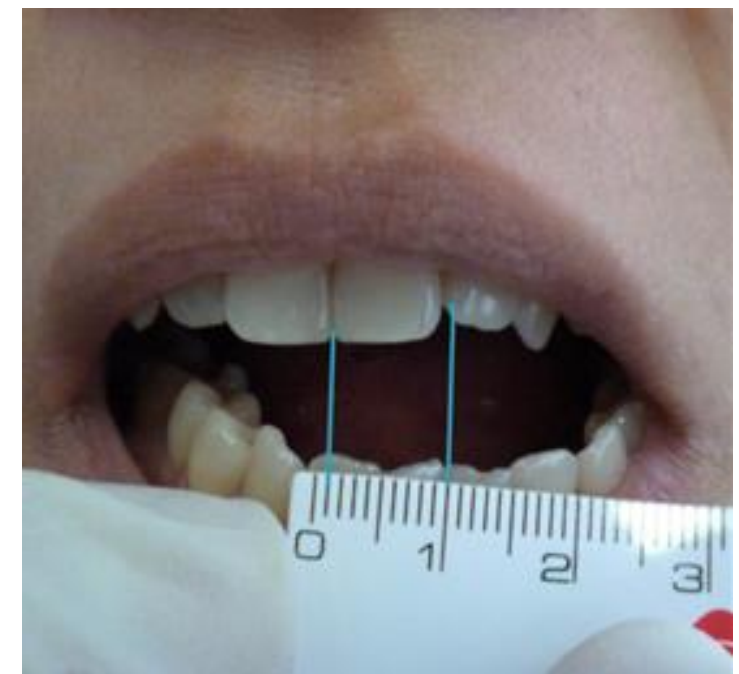

Figura 13: Medida de lateralidade esquerda (9mm) após duas semanas de tratamento

A paciente foi encaminhada para a especialização de Ortodontia da UNICSUL-Manaus para avaliação ortodôntica. Também foi solicitado à paciente que procurasse um fonoaudiólogo, já que os tratamentos de DTMs são multidisciplinares.

\section{DISCUSSÃO}

Os tratamentos que têm sido sugeridos para as desordens temporomandibulares, em especial para o mioespasmo, variam entre um grande espectro de modalidades.

Nicolakis et al. ${ }^{16}$ relatam que os exercícios terapêuticos, especificamente os exercícios ativos e passivos dos movimentos mandibulares, juntamente com exercícios de relaxamento e correção postural, são úteis no tratamento da dor e na incapacidade funcional em pacientes com DTM.

Kogawa et al. ${ }^{17}$ destacam a importância do fisioterapeuta na abordagem terapêutica multidisciplinar da DTM, contribuindo para o alívio da dor musculoesquelética, reduzindo a inflamação e restaurando a função motora normal.

Cerca de $37,5 \%$ da população apresentam ao menos um sintoma de $\mathrm{DTM}^{31}$. Entre estudantes universitários, estima-se que $41,3 \%$ a $68,6 \%$ apresentem algum sinal ou sintoma de $\mathrm{DTM}^{18,19}$. O caso clínico relatado no presente trabalho corrobora essas afirmativas pois trata-se de paciente universitária com ao menos um sinal de DTM, ou seja, apresentava mioespasmo.

Revisão sistemática com metanálise conduzida por $\mathrm{Al}-$ Jundi et al. $^{20}$ estimou prevalência de necessidade de tratamento da DTM na população adulta em 15,6\%, sendo que as estimativas para a população jovem, de 19 a 45 anos, foi maior do que para a de adultos acima de 46 anos $^{34}$. Outrossim, segundo Biasotto ${ }^{21}$ as mulheres são mais atingidas do que os homens no desenvolvimento de distúrbios da articulação temporomandibular.

O relato de caso descrito no presente trabalho é concordante com Al-Jundi et al. ${ }^{20}$ e Biasoto $^{21}$, já que se trata de paciente do sexo feminino, adulta, na faixa etária de 19 a 45 anos (33 anos) e que sentiu necessidade de procurar tratamento da DTM.

Magnusson et $\mathrm{al}^{22}$ observaram benefícios significativos no alívio da dor e disfunção quando o exercício fisioterapêutico foi associado à placa miorrelaxante em pacientes com DTM. Os resultados obtidos no presente trabalho também corroboram os benefícios dessa terapêutica, pois a paciente teve melhora expressiva, com aumento de 8 mm da amplitude da lateralidade esquerda.

\section{CONCLUSÃO}

A etiologia dos mioespasmos não tem sido bem documentada. É provável que muitos fatores se combinem para promover o mioespasmo, como as condições musculares locais, dores de cabeça e de ouvido, dor facial, má oclusão aguda dentre outros. Compete aos cirurgiões - dentistas tratálos em conjunto com outras especialidades.

\section{REFERÊNCIAS}

1. Manganello LCS, Silveira ME, Silva AAF. Cirurgia da articulação Temporomandibular. São Paulo: Santos; 2014.

2. Okeson JP. Tratamento das Desordens Temporomandibulares e Oclusão, 7. ed. Rio de Janeiro: Elsevier; 2013.

3. Siqueira JTT, Texeira MJ e colaboradores. Dores Orofaciais. Diagnóstico e Tratamento. São Paulo: Artes Médicas; 2012.

4. Lund JP, Widmer CG. Evaluation of the use of surface electromyography in the diagnosis, documentation, and treatment of dental patients. J Craniomandib Disord. 1989; 3(3):125-37

5. Curran SL, Carlson CR, Okeson JP. Emotional and physiologic responses to laboratory challenges: patients with temporomandibular disorders versus matched control subjects. J Orofac Pain, 1996; 10(2):141-50

6. Yemm R. A neurophysiological approach to the pathology and etiology of temporomandibular dysfunction, J Oral Rehabil. 1985; 12(4):343-53

7. Lund JP, Donga R, Widmer CG, Stohler CS. The painadaptation model: a discussion of the relationship between chronic musculoskeletal pain and motor activity. Can J Physiol Pharmacol. 1991; 69(5):683-94

8. Carlson CR, Okeson JP, Falace DA, Nitz AJ, Lindroth JE. Reduction of pain and EMG activity in the masseter 
region by trapezius trigger point injection. Pain. 1993 55(3):397-400

9. Okeson JP. Tratamento das Desordens Temporomandibulares e Oclusão, 6. ed. Rio de Janeiro: Elsevier; 2008.

10. Okeson JP. Dores Bucofaciais de Bell. 5. ed. São Paulo: Quintessence Publishing; 1998.

11. Wright EF. Manual of temporomandibular disorders. Iowa: Blackwell-Munksgaard; 2005.

12. Neto AO, Issy AM, Siqueira JTT, Texeira MJ e colaboradores. Dor: princípios e prática. Porto Alegre: Artmed; 2009.

13. Cohen S, Hargreaves KM. Caminhos da Polpa. 10. ed. Rio de Janeiro: Elsevier; 2011.

14. American Academy of Orofacial Pain, with Okeson JP (ed). Orofacial Pain: Guidelines for Assessment, Diagnosis and Management. Chicago: Quintessence; 1996.

15. Okeson JP. Management of Temporomandibular Disorders and Occlusion, 5. ed. St Louis: CV Mosby; 2003.

16. Nicolakis P, ErdogmusB, Kopf A, Djaber-Ansari A, Pieshlinger E, Fialka-Moser V. Exercice therapy for craniomandibular disorders. Arch Phys Med Reabil. 2000; 81(9):1137-42.

17. Kogawa EM, Kato MT, Santos CN, Conti PCR. Evaluation of the efficacy of low-level laser therapy (LLLT) and the microelectric neurostimulation (MENS) in the treatment of myogenic temporomandibular disorders: a randomized clinical trial. J Appl Oral Sci. 2005; 13(3):280-5

18. Bonjardim LR, Lopes-Filho RJ, Amado G, Albuquerque RL Jr, Gonçalves SR. Association between symptoms of temporomandibular disorders and gender, morphological occlusion and psychological factors in a group of university students. Indian J Dent Res. 2009; 20(2):190-4.

19. Pedroni CR, de Oliveira AS, Guaratini MI. Prevalence study of signs and symptoms of temporomandibular disorders in university students. $\mathbf{J}$ Oral Rehabil. 2003;30(3):283-9

20. Al-Jundi MA, John MT, Setz JM, Szentpétery A, Kuss O. Meta-analysis of treatment need for temporomandibular disorders in adult nonpatients. J Orofac Pain. 2008; 22(2): $97-107$

21. Biasotto GDA. Abordagem interdisciplinar das disfunções temporomandibulares. São Paulo: Manole; 2005 .

22. Magnusson T, Egermark I, Carlsson GE. Treatment received, treatment demand, and treatment need for temporomandibular disorders in 35-year-old subjects. Cranio. 2002; 20(1):11-7

\section{CONFLITO DE INTERESSES}

Os autores declaram não haver conflitos de interesse.

\section{AUTOR PARA CORRESPONDÊNCIA}

Helinaldo Corrêa da Conceição

helinadouea@gmail.com

Submetido em 18/01/2018

Aceito em 16/02/2018 